\title{
Feasibility of Using Masticatory Muscles to Assess Skeletal Muscle Mass in Patients with Oral Cavity Cancer
}

\section{Sheng-Wei Chang}

Chiayi Chang Gung Memorial Hospital

\section{Cheng-Ming Hsu}

Chiayi Chang Gung Memorial Hospital

\section{Yuan-Hsiung Tsai}

Chiayi Chang Gung Memorial Hospital

\section{Geng-He Chang}

Chiayi Chang Gung Memorial Hospital

\section{Ming-Shao Tsai}

Chiayi Chang Gung Memorial Hospital

\section{Ethan I. Huang}

Chiayi Chang Gung Memorial Hospital

\section{Ku-Hao Fang}

Chang Gung Memorial Hospital

\section{Kai-Ping Chang}

Chang Gung Memorial Hospital

Yao-Te Tsai ( $\square$ yaote1215@gmail.com)

Chiayi Chang Gung Memorial Hospital https://orcid.org/0000-0002-2313-0890

\section{Research article}

Keywords: oral cavity cancer, sarcopenia, low skeletal muscle mass, computed tomography, image analysis

Posted Date: August 3rd, 2019

DOI: https://doi.org/10.21203/rs.2.12373/v1

License: (c) (1) This work is licensed under a Creative Commons Attribution 4.0 International License.

Read Full License 


\section{Abstract}

Background Sarcopenia is typically assessed using abdominal computed tomography (CT) scans, which are not obtained routinely in patients with oral cavity cancer. In order to investigate the feasibility of using masticatory muscles to assess general skeletal muscle mass (SMM) in oral cancer patients, we aimed to correlate the masticatory SMM evaluated on head and neck CT-images with the SMM measurement at the level of L3 on abdominal CT-scans. Methods The abdominal and head and neck CT-scans of the oral cavity squamous cell carcinoma (OSCC, $n=54)$ and trauma $(n=50)$ patients were analyzed retrospectively. All muscles at the L3 level were delineated on abdominal CT scans, and skeletal muscles at the mandibular notch level and C3 level were delineated on head and neck CT scans. Cross-sectional area (CSA) of the muscles at the mandibular notch and C3 level were compared to skeletal muscle CSA at the L3 level using linear regression, and a multivariate linear regression model was also calculated. Results The OSCC patients had significantly smaller muscle CSA at the mandibular notch level than trauma patients (1221.4 vs. $1512.2 \mathrm{~mm} 2, \mathrm{p}=0.006$ ). Masticatory muscle CSA strongly predicted the $\mathrm{L} 3$ muscle CSA ( $r=0.880, p<0.001)$, and this correlation was stronger in the multivariate model including age, body weight, and sex $(r=0.898, p<0.001)$. Conclusions Evaluation of the masticatory skeletal muscle mass on head and neck CT images is simple, feasible, and may be an alternative to abdominal CT scans. Assessment of masticatory muscle at mandibular notch can be performed using routinely obtained scans without additional imaging or cost. Identifying patients at risk of being sarcopenic may select oral cancer patients for nutritional intervention and appropriate treatment modality to improve their prognosis.

\section{Background}

Poor nutritional state is a common issue in patients with cancer and results in loss of skeletal muscle mass (SMM), the largest component of lean body mass, whereas body fat may still be preserved $[1,2]$. Changes in SMM and physical performance may predate clinically apparent cachexia, and patients with head and neck cancer (HNC) reportedly have a higher risk of muscle wasting compared with patients with other malignancies $[3,4]$. Sarcopenia is defined as the combination of generalized and progressive loss of SMM with either poor physical performance or low muscle strength[5]. The loss of SMM and decreased physical capability is reportedly associated with adverse prognosis in various malignancies, including $\mathrm{HNC}[2,4,6-9]$. The literature reveals that sarcopenia in patients with cancer is not only associated with postoperative complications $[10,11]$ and toxicity of adjuvant therapy[11-14], but also adversely affects the cancer prognosis [15]. Therefore, the measurement of SMM for the assessment of sarcopenia in patients with $\mathrm{HNC}$ is of great importance to clinical practice and oncology treatment planning.

Recently, SMM has widely been researched in cancer patients, using a measurement of skeletal muscle cross-sectional area (CSA) at the level of L3 on computed tomography (CT) or positron emission tomography (PET)-CT scans as an indicator of total SMM[16]. Even though patients with HNC are prone to sarcopenic status, abdomen CT imaging or PET-CT scan are not routinely performed in patients with 
HNC, making the SMM measurements at the L3 level not always be applicable. Swartz et al[16] proposed that assessing C3 muscle CSA on a head and neck CT scan could be an excellent alternative to abdominal CT scans [17]. Nevertheless, neck lymphadenopathy or locally advanced disease may hinder interpretation of the C3 muscle CSA in HNC patients.

In HNC patients, the sarcopenia reflect not only the swallowing function but also the nutritional status [18]. The masticatory muscles were also revealed to be equally valid marker of sarcopenia in trauma patients $[19,20]$. However, the correlation between masticatory muscle and underlying sarcopenia has never been studied in HNC patients. Herein, we aimed to investigate whether masticatory muscle mass may be evaluated on a conventional head and neck CT-scan and correlated with sarcopenia in oral cavity squamous cell carcinoma (OSCC) patients by comparing the masticatory muscle CSAs at the level of mandibular notch to L3 muscle measurement. If the masticatory muscles CSA on head and neck CT-scan could be a reliable alternative to determine the SMM, it may be a cost-effective and more readily available tool to assess sarcopenia in OSCC patients.

\section{Methods}

This study enrolled 54 randomly chosen OSCC patients undergoing a total-body (PET-) CT-scan as a part of tumor staging and curative surgery at Chiayi Chang Gung Memorial Hospital from January 1, 2014, to December 31, 2018. Additionally, 50 trauma patients without cancer history who underwent whole-body CT scan within 2018 were randomly selected as healthy controls. Age, sex, body weight, and body mass index (BMI) were documented for all participants, and pathological AJCC TNM staging (7th edition) was recorded for OSCC patients. This study was approved by the Institutional Review Board of Chang Gung Memorial Hospital.

All images were obtained from the picture archiving and communication system of the Chiayi branch of Chang Gung Memorial Hospital by using DICOM 3.0 files. The patients were scanned using the CT scanner: Toshiba Aquilion 64 (Canon Medical System Corporation, Otawara, Tochigi, Japan) with parameters: $120 \mathrm{kV}, 250 \mathrm{~mA}$, slice thickness $5 \mathrm{~mm}$, slice interval $5 \mathrm{~mm}$, pitch 1.312 for both abdomen and head and neck regions. Muscle CSAs were measured using the ImageJ v1.52a freeware package (Wayne Rasband, National Institutes of Health, USA) with threshold selection from -29 to $150 \mathrm{HU}[16]$ as shown in Fig. 1. The mandibular notch was chosen as the reference point for the measurement of the CSA of the masticatory muscles in the head and neck CT scans. We chose this level because the notch is easily identifiable on a CT scan, and both the CSAs of the masseter muscles (lateral to the mandibular notch) and pterygoid muscles (medial to the mandibular notch) can be assessed in the same slice. Image selection was performed using the standard procedure of scrolling through the ramus of the mandibular bone in a caudad-to-cephalad direction, and the first CT slide to show the mandibular notch entirely was selected.

Image selection and analysis of the cervical (C3) and lumbar (L3) vertebra were performed as per previously published reports $[16,17,21]$. CSAs of the paravertebral muscles (PVM) and the 
sternocleidomastoid muscles (SCM) at the C3 level were measured, and the sum of these muscles was defined as the $\mathrm{C} 3$ muscles. The sum of masticatory muscles at the mandibular notch level and all skeletal muscles at L3 level were also obtained (Fig. 1). Delineation of these muscles was performed manually by two independent board-certified radiologists (S. W. Chang and W. S. Tsai) with a doubleblinded method, and all clinical characteristics were hidden. Both the radiologists used manual delineation and threshold selection to delineate all head and neck (masticatory and C3 muscles) scans first and then delineated all abdominal (L3) scans. After delineation, the CSA of the delineated area was automatically retrieved as the total sum of the delineated pixels. The CSA of pixels that had a radiodensity between -29 to $150 \mathrm{HU}$ was also retrieved. Both approaches were performed to evaluate whether the target muscle CSAs were similar between manual delineation and thresholding selection with radiodensity. Intraclass correlation (ICC) was conducted to assess interobserver reliability using a twoway mixed model.

The characteristics and CSAs of the two patient groups were compared using independent-samples $t$ tests for normally distributed variables, independent-samples median tests for skewed variables, and Fisher exact tests for categorical variables. Normality testing was performed using the KolmogorovSmirnov test. Pearson correlation coefficients were calculated between the measurements of the CSAs of the masticatory muscles at the mandibular notch level, C3 and L3 muscles. Linear regression analysis was used to examine the association between (1) the CSAs of the masticatory and L3 muscles and (2) the CSAs of the C3 and L3 muscles. The statistical significance was set at $p<0.05$. The similarity of the association for the OSCC and trauma patients was investigated by using the variable "groups" as an interaction term in the statistical analysis. For the multivariate analysis model, all demographic data, C3 level muscle and masticatory muscle were selected. Backward selection approach was performed and the variables are discarded if $p$ value higher than 0.1 . All analyses were performed in using SAS Enterprise Guide version 6.3.

\section{Results}

Table 1 lists the patient characteristics, and 50 trauma patients and 54 OSCC patients were enrolled. The groups were comparable in sex distribution, body weight and BMI. The median age was significantly higher in OSCC patients $(p=0.014)$. Of the OSCC patients, thirty patients $(55.6 \%)$ had stages I and II disease, and twenty-four patients (44.4\%) had stages III and IV disease. Nine patients $(16.7 \%)$ had cervical lymph nodes metastasis ( $\mathrm{N}+$ disease). Interobserver reliability was assessed and revealed high agreement between both observers at all the CSAs (ICC $=0.99$ for L3 PVM, $p<0.001$; ICC $=0.991$ for C3 muscle, $p<0.001$; and ICC 0.997 for the masticatory muscles, $p<0.001$ ). With almost perfect agreement between both observers, all the muscle CSA calculations for the final analysis were based on the measurements of the main observer (S. W. Chang).

When comparing the manually delineated area to the radiodensity selected muscle area, there were consistent results in masticatory muscles, including masseter and pterygoid muscles (Table 2). For trauma patients, the masticatory muscles were $1523.4 \mathrm{~mm}^{2}$ (manual) and $1512.2 \mathrm{~mm}^{2}$ (threshold) with $p$ 
$=0.92$. For OSCC patients, the masticatory muscles was $1207.8 \mathrm{~mm}^{2}$ (manual) and $1221.4 \mathrm{~mm}^{2}$ (threshold) with $p=0.91$. The manually delineated area of the C3 and L3 muscles show significant overestimation compared to the threshold selection area $(7.27 \%, p=0.008$ and $6.05 \%, p=0.011$ respectively, Table 2). Because of the overestimation, potentially resulted from the muscle fattening, we used the threshold selected muscle area of C3 and L3 muscles in the further analysis. The sum of the CSA of the masticatory muscles at mandibular notch, as well as the CSA of the masseter and pterygoid muscles were significantly lower in the OSCC group compared to the trauma group (Table 3, corrected for age and sex). By contrast, the C3 and L3 muscle CSAs exhibited no statistical significance between the patients with trauma and OSCC.

Because there was no apparent intergroup interaction, the patients from the oral cancer and trauma groups could be analyzed as a single entity. Fig. 2 shows the correlation between masticatory muscles at the mandibular notch and L3 muscle measurements. Pearson correlation revealed significant correlation between the masticatory and L3 muscles $(r=0.880, p<0.001)$ and the C3 and L3 muscles $(r=0.948, p<$ 0.001). The following prediction formulas were thus established because of the high correlations:

L3 CSA $\left(\mathrm{mm}^{2}\right)=5294+4.03 \times$ masticator CSA $\left(\mathrm{mm}^{2}\right)$

L3 CSA $\left(\mathrm{mm}^{2}\right)=5357+2.29 \times \mathrm{C} 3 \mathrm{CSA}\left(\mathrm{mm}^{2}\right)$

Furthermore, several clinical variables were introduced into the regression model. Cancer status, age, sex, weight, and BMI were significant covariates, apart from masticatory CSA. All the factors were backward eliminated and showed $p<0.1$ in the multivariate model, except for the variable of cancer status, which was removed during the process. A multivariate model was established that can be depicted with the following formula:

L3 CSA $\left(\mathrm{mm}^{2}\right)=4704+3.9 \times$ masticator CSA $\left(\mathrm{mm}^{2}\right)+111 \times$ weight $(\mathrm{kg})-34.7 \times$ age (years) $-155.9 \times$ sex $($ female $=1$, male $=0), r=0.898$

Additional analyses within the OSCC patient group revealed no significant differences in skeletal muscle CSA measured at the mandibular notch, C3, or L3 levels between patients with and without neck metastatic lymphadenopathy. Similarly, there were no significant differences in these characteristics between the patients with T1-2 versus T3-4 disease.

\section{Discussion}

Patients with oral cavity cancer are at an elevated risk of developing dysphagia, malnutrition, and subsequently sarcopenia than patients with other types of malignancy[19]. However, performing abdominal CT scans to evaluate sarcopenia in patients with oral cancer would generate additional costs and increase radiation exposure. We therefore investigated whether masticatory muscle CSA on a head and neck CT-scan correlates with L3 muscle CSA on abdominal CT-scans and observed a good correlation between the two with consistent interobserver agreement. To the best of our knowledge, this is 
the first study to assess the correlation between masticatory SMM at the mandibular notch level and SMM measurement at the level of L3. This correlation positively suggests that CT scan of the head and neck region may also be used to evaluate the L3 muscle CSA and to determine the presence of sarcopenia.

The clinical significance and high percentage of sarcopenia in HNC patients has been highlighted in recent studies, demonstrating strong associations with treatment toxicity and poor survival[22]. Wendrich et al indicated that the presence of sarcopenia among patients undergoing chemoradiation for locally advanced head and neck cancer increased both the risk of developing, and the severity of, treatmentrelated toxicities[14]. Recent studies also found that HNC patients with low skeletal muscle mass, as measured by CT scans at presentation or after radiotherapy, exhibit decreased overall and cancer specific survival[2, 23]. Furthermore, HNC patients treated with definitive RT and are compliant with regular dietetic counseling appear to have improved oncologic outcomes[22] Nishikawa et al. investigated the effect of sarcopenia in eighty-five patients with HNC receiving surgery and radiotherapy, and proposed that sarcopenic patients will benefit more from surgery than radiotherapy[4]. Although sarcopenia and malnutrition are emerging issues in HNC patients, there is no information available on the prevalence and impact of sarcopenia in patients with oral cavity cancer. In this study, we observed that masticatory SMM was significantly lower in OSCC than in trauma patients, and the C3 and L3 muscle CSAs were also smaller in OSCC patients without statistical significance. Therefore, we suggested that sarcopenia may be highly prevalent in patients with oral cavity cancer, and the masticatory muscle may be more representative to sarcopenia than C3 and L3 muscle in OSCC patients. This may be explained by malnutrition and cancer induced muscle wasting, as the oral cancer may impair masticatory and swallowing function with higher muscle oxidative metabolism[24].

Perhaps the sarcopenic OSCC patients may benefit from interventions such as additional nutritional supplement to improve their oncologic outcomes. In the future, OSCC patients at high risk of adverse outcomes related to low SMM or sarcopenia might benefit from individualized treatment plan or additional supportive therapy. Several possible interventions, such as pre-habilitation before surgery[25], altered chemotherapy dosing[26], optimal perioperative care and enhanced recovery after surgery[27], progressive physiotherapy[28], protein- and energy-dense nutritional support may be considered[29].

Correlation of muscle mass to cancer stages is also of interest. In our cohort, we found no statistically significant difference in SMM in the OSCC patients with advanced T- or N-classifications. However, the lack of statistical significance may be owing to the relatively small sample size of this study. A prospective study with a larger and more homogeneous cohort should be performed in the future to validate the present findings and evaluate the prognostic significance of masticatory muscle mass in patients with OSCC. The mandibular notch was chosen as the reference point in the head and neck CT scan because the notch is not only an well identifiable landmark but also a surgical landmark with prognostic significance[30]. Our colleagues advocated that infra-notch T4b oral cavity cancer is resectable and has comparable outcomes to the T4a disease[31]. The masticatory muscles at the 
mandibular notch level can therefore be clearly defined, and we found the masticatory SMM can be measured in a single slice of head and neck CT scan with excellent interobserver agreement.

We investigated whether manual delineation of muscles alone is comparable to threshold selection of skeletal muscle cross section area using a window of -29 to $+150 \mathrm{HU}$. We found that manual delineation resulted in high overestimation of C3 and L3 muscle mass compared to automatic identification of SMM. By contrast, the masticatory muscles mass at notch level showed consistent results in both manually delineated area and radiodensity selected area. This may be explained by the less anatomical complexity of masticatory muscles at notch level than muscles at C3 and L3 levels. Besides, the SMM measurements at C3 and L3 levels involving delineating sophisticate region of interest and need third party programs to achieve. By comparison, masticatory SMM at notch level is more uniform in shape and can be measured directly on most of picture archiving and communicating system (PACS). Based on these factors, we propose that assessment of sarcopenia on head and neck CT scans is easier and more robust by measuring masticatory muscle CSA at the mandibular notch level than C3 muscles.

Recently, Swartz et al. suggested the use of CSAs of the neck muscles at the C3 level to investigate the correlation of SMM on head and neck CT scans and abdominal CT imaging. However, a major limitation of their technique is the frequent hindrance to cervical muscle assessment because of the presence of local-regional tumor extension or metastatic lymphadenopathy-present in approximately $57 \%$ of patients with $\mathrm{HNC}$ at the time of diagnosis[32]. Furthermore, SCM muscle assessment is particularly risky because of the lymph node stations abutting closely to this muscle[33]. In current study, patients with resectable OSCC usually presented without supra-notch invasion. Therefore, assessment of the masticatory muscles at the mandibular notch level will not be impaired by the presence of primary tumor invasion or metastatic lymphadenopathy. Moreover, Swartz et al. enrolled a study population that was more than $70 \%$ patients with oropharyngeal cancer, whereas cases of OSCC comprised less than $10 \%$ of the total number of patients. Our study further extended the knowledge regarding measuring skeletal muscle CSA on head and neck CT scans as an assessment of sarcopenia to patients with OSCC.

This study has several limitations. As with all the retrospective studies, heterogeneous patient populations preclude overgeneralization of our study results. Besides, not all OSCC patients receive staging and follow-up PET-CTs, so some bias in sampling is possible. To investigate whether patient selection would indeed result in biased results, we also included trauma patients who would otherwise be considered healthy controls. Because there was no difference in the correlation between masticatory muscle and L3 muscle CSA between OSCC and trauma groups, our results may be deduced to both oral cancer patients and healthy individuals. Finally, even though CSA assessment at the mandibular notch and C3 level by using a single slice has proved to be one method for estimating sarcopenia, estimation of SMM by using the volume data is considered the standard[16, 17, 21]. Therefore, the masticatory muscle assessment in the future studies should be assessed by summation of all the CSA slices and estimation of their volumes rather than just CSA measurement [34].

\section{Conclusions}


In OSCC patients, the measurement of masticatory SMM at the level of mandibular notch provides a reliable and robust alternative to SMM measurement at the level of $L 3$, based on the strong correlation between skeletal muscle CSA at mandibular notch and L3 level. This novel approach provides clinicians with a cost-effective screening tool for skeletal muscle mass and sarcopenia in the vast majority of OSCC patients, and allows for retrospective and prospective research into the prognostic value of low masticatory SMM without additional imaging or cost.

\section{List Of Abbreviations}

OSCC: oral cavity squamous cell carcinoma

SMM: skeletal muscle mass

HNC: head and neck cancer

CT: computed tomography

PET: positron emission tomography

CSA: cross-sectional area

PVM: paravertebral muscles

SCM: sternocleidomastoid muscles

PACS: picture archiving and communicating system

BMI: body mass index

ICC: Intraclass correlation

\section{Declarations}

\section{Ethics approval and consent to participate}

This study was approved by the Institutional Review Board of Chang Gung Memorial Hospital.

The informed consent was written and obtained from all study participants.

\section{Consent for publication}

Not applicable.

\section{Availability of data and materials}


The datasets generated and/or analyzed during the current study are not publicly available due to the personal information protection act from Taiwan government but are available from the corresponding author on reasonable request.

\section{Competing interests}

The authors declare that they have no competing interest.

\section{Funding}

Not applicable.

\section{Authors' contributions}

SW: drafted the manuscript

CM: analyzed the patients data

$\mathrm{YH}$ : interpreted the patients data

$\mathrm{GH}$ : designed the study

MS: performed statistical analysis

El: interpretation of data

$\mathrm{KH}$ : imaging data collection and analysis

KP: study design

YT: substantively revise the article

All authors read and approved the final manuscript."

\section{Acknowledgements}

This manuscript was edited by Wallace Academic Editing.

\section{References}

[1] Prado CMM, Lieffers JR, McCargar LJ, Reiman T, Sawyer MB, Martin L, et al. Prevalence and clinical implications of sarcopenic obesity in patients with solid tumours of the respiratory and gastrointestinal tracts: a population-based study. The Lancet Oncology. 2008;9:629-35. 
[2] Grossberg AJ, Chamchod S, Fuller CD, Mohamed AS, Heukelom J, Eichelberger H, et al. Association of Body Composition With Survival and Locoregional Control of Radiotherapy-Treated Head and Neck Squamous Cell Carcinoma. JAMA Oncol. 2016;2:782-9.

[3] Pressoir M, Desne S, Berchery D, Rossignol G, Poiree B, Meslier M, et al. Prevalence, risk factors and clinical implications of malnutrition in French Comprehensive Cancer Centres. British journal of cancer. 2010;102:966-71.

[4] Nishikawa D, Hanai N, Suzuki H, Koide Y, Beppu S, Hasegawa Y. The Impact of Skeletal Muscle Depletion on Head and Neck Squamous Cell Carcinoma. ORL; journal for oto-rhino-laryngology and its related specialties. 2018;80:1-9.

[5] Cruz-Jentoft AJ, Baeyens JP, Bauer JM, Boirie Y, Cederholm T, Landi F, et al. Sarcopenia: European consensus on definition and diagnosis: Report of the European Working Group on Sarcopenia in Older People. Age and ageing. 2010;39:412-23.

[6] Go SI, Park MJ, Song HN, Kang MH, Park HJ, Jeon KN, et al. Sarcopenia and inflammation are independent predictors of survival in male patients newly diagnosed with small cell lung cancer. Support Care Cancer. 2016;24:2075-84.

[7] Mei KL, Batsis JA, Mills JB, Holubar SD. Sarcopenia and sarcopenic obesity: do they predict inferior oncologic outcomes after gastrointestinal cancer surgery? Perioper Med (Lond). 2016;5:30.

[8] Iritani S. Skeletal muscle depletion is an independent prognostic factor for hepatocellular carcinoma. J Gastroenterol. 2014.

[9] Psutka SP, Boorjian SA, Moynagh MR, Schmit GD, Costello BA, Thompson RH, et al. Decreased Skeletal Muscle Mass is Associated with an Increased Risk of Mortality after Radical Nephrectomy for Localized Renal Cell Cancer. J Urol. 2016;195:270-6.

[10] Harimoto N, Shirabe K, Yamashita YI, Ikegami T, Yoshizumi T, Soejima Y, et al. Sarcopenia as a predictor of prognosis in patients following hepatectomy for hepatocellular carcinoma. The British journal of surgery. 2013;100:1523-30.

[11] Lieffers JR, Bathe OF, Fassbender K, Winget M, Baracos VE. Sarcopenia is associated with postoperative infection and delayed recovery from colorectal cancer resection surgery. British journal of cancer. 2012;107:931-6.

[12] Gregoire V, Lefebvre JL, Licitra L, Felip E, Group E-E-EGW. Squamous cell carcinoma of the head and neck: EHNS-ESMO-ESTRO Clinical Practice Guidelines for diagnosis, treatment and follow-up. Annals of oncology: official journal of the European Society for Medical Oncology. 2010;21 Suppl 5:v184-6.

[13] Pignon JP, le Maitre A, Maillard E, Bourhis J, Group M-NC. Meta-analysis of chemotherapy in head and neck cancer (MACH-NC): an update on 93 randomised trials and 17,346 patients. Radiotherapy and 
oncology: journal of the European Society for Therapeutic Radiology and Oncology. 2009;92:4-14.

[14] Wendrich AW, Swartz JE, Bril SI, Wegner I, de Graeff A, Smid EJ, et al. Low skeletal muscle mass is a predictive factor for chemotherapy dose-limiting toxicity in patients with locally advanced head and neck cancer. Oral oncology. 2017;71:26-33.

[15] Prado CM, Baracos VE, McCargar LJ, Reiman T, Mourtzakis M, Tonkin K, et al. Sarcopenia as a determinant of chemotherapy toxicity and time to tumor progression in metastatic breast cancer patients receiving capecitabine treatment. Clinical cancer research: an official journal of the American Association for Cancer Research. 2009;15:2920-6.

[16] Swartz JE, Pothen AJ, Wegner I, Smid EJ, Swart KM, de Bree R, et al. Feasibility of using head and neck CT imaging to assess skeletal muscle mass in head and neck cancer patients. Oral oncology. 2016;62:28-33.

[17] Bril SI, Wendrich AW, Swartz JE, Wegner I, Pameijer F, Smid EJ, et al. Interobserver agreement of skeletal muscle mass measurement on head and neck CT imaging at the level of the third cervical vertebra. Eur Arch Otorhinolaryngol. 2019.

[18] Saitoh M, Ishida J, Konishi M, Springer J. The concept that focuses on oral motor and feeding function in cancer patients with muscle wasting: Skeletal muscle mass is associated with severe dysphagia in cancer patients. J Cachexia Sarcopenia Muscle. 2016;7:233-4.

[19] Wallace JD, Calvo RY, Lewis PR, Brill JB, Shackford SR, Sise MJ, et al. Sarcopenia as a predictor of mortality in elderly blunt trauma patients: Comparing the masseter to the psoas using computed tomography. The journal of trauma and acute care surgery. 2017;82:65-72.

[20] Hu P, Uhlich R, White J, Kerby J, Bosarge P. Sarcopenia Measured Using Masseter Area Predicts Early Mortality following Severe Traumatic Brain Injury. Journal of neurotrauma. 2018;35:2400-6.

[21] Mourtzakis M, Prado CM, Lieffers JR, Reiman T, McCargar LJ, Baracos VE. A practical and precise approach to quantification of body composition in cancer patients using computed tomography images acquired during routine care. Appl Physiol Nutr Metab. 2008;33:997-1006.

[22] Kabarriti R, Bontempo A, Romano M, McGovern KP, Asaro A, Viswanathan S, et al. The impact of dietary regimen compliance on outcomes for HNSCC patients treated with radiation therapy. Support Care Cancer. 2018;26:3307-13.

[23] Tamaki A, Manzoor NF, Babajanian E, Ascha M, Rezaee R, Zender CA. Clinical Significance of Sarcopenia among Patients with Advanced Oropharyngeal Cancer. Otolaryngology-head and neck surgery: official journal of American Academy of Otolaryngology-Head and Neck Surgery. 2018:194599818793857. 
[24] Carson JA, Hardee JP, VanderVeen BN. The emerging role of skeletal muscle oxidative metabolism as a biological target and cellular regulator of cancer-induced muscle wasting. Semin Cell Dev Biol. 2016;54:53-67.

[25] Huang J, Lai Y, Zhou X, Li S, Su J, Yang M, et al. Short-term high-intensity rehabilitation in radically treated lung cancer: a three-armed randomized controlled trial. J Thorac Dis. 2017;9:1919-29.

[26] Sjoblom B, Benth JS, Gronberg BH, Baracos VE, Sawyer MB, Flotten O, et al. Drug Dose Per Kilogram Lean Body Mass Predicts Hematologic Toxicity From Carboplatin-Doublet Chemotherapy in Advanced Non-Small-Cell Lung Cancer. Clin Lung Cancer. 2017;18:e129-e36.

[27] Dort JC, Farwell DG, Findlay M, Huber GF, Kerr P, Shea-Budgell MA, et al. Optimal Perioperative Care in Major Head and Neck Cancer Surgery With Free Flap Reconstruction: A Consensus Review and Recommendations From the Enhanced Recovery After Surgery Society. JAMA Otolaryngol Head Neck Surg. 2017;143:292-303.

[28] Lonbro S, Dalgas U, Primdahl H, Overgaard J, Overgaard K. Feasibility and efficacy of progressive resistance training and dietary supplements in radiotherapy treated head and neck cancer patients-the DAHANCA 25A study. Acta Oncol. 2013;52:310-8.

[29] Weed HG, Ferguson ML, Gaff RL, Hustead DS, Nelson JL, Voss AC. Lean body mass gain in patients with head and neck squamous cell cancer treated perioperatively with a protein- and energy-dense nutritional supplement containing eicosapentaenoic acid. Head Neck. 2011;33:1027-33.

[30] Wakabayashi H, Matsushima M, Uwano R, Watanabe N, Oritsu H, Shimizu Y. Skeletal muscle mass is associated with severe dysphagia in cancer patients. J Cachexia Sarcopenia Muscle. 2015;6:351-7.

[31] Liao CT, Chang JT, Wang HM, Ng SH, Hsueh C, Lee LY, et al. Surgical outcome of T4a and resected T4b oral cavity cancer. Cancer. 2006;107:337-44.

[32] R. L. Distribution of cervical lymph node metastases from squamous cell carcinoma of the upper respiratory and digestive tracts. Cancer. 1972;Jun;29(6):4.

[33] K. Thomas Robbins MARS, MD; Jesus E. Medina, MD; Joseph A. Califano, MD; Gregory T. Wolf MAF, MD; Peter M. Som, MD; Terry A. Day, MD. Consensus Statement on the Classification and Terminology of Neck Dissection. ARCH OTOLARYNGOL HEAD NECK SURG. 2008;VOL 134 (NO. 5):3.

[34] von Haehling S, Morley JE, Coats AJS, Anker SD. Ethical guidelines for publishing in the Journal of Cachexia, Sarcopenia and Muscle: update 2017. J Cachexia Sarcopenia Muscle 2017;8: 1081-1083

\section{Tables}

Table 1. Baseline demographics comparison of the patients with trauma and oral cancer. 


\begin{tabular}{|c|c|c|c|}
\hline Variable & Trauma $(n=50)$ & Oral cancer $(n=54)$ & $p$-value \\
\hline \multicolumn{4}{|l|}{ Sex } \\
\hline Female & $11(22.0)$ & $4(7.4)$ & 0.09 \\
\hline Male & $39(78.0)$ & $50(92.6)$ & 0.43 \\
\hline Age & $59.6(9.9)$ & $74.2(6.5)$ & 0.014 \\
\hline Weight & $68.1(15.6)$ & $64.8(11.9)$ & 0.22 \\
\hline BMI & 25.2 (4.1)\# & 24.4 (4.7)\# & 0.35 \\
\hline Cancer stage & n.a. & & n.a. \\
\hline Early $(\mathrm{I}, \mathrm{II})$ & & $30(55.6)$ & \\
\hline Advanced (III,IV) & & $24(44.4)$ & \\
\hline T classification & n.a. & & n.a. \\
\hline Т 1-2 & & $25(46.3)$ & \\
\hline Т 3-4 & & $29(53.7)$ & \\
\hline $\mathrm{N}$ classification & n.a. & & n.a. \\
\hline NO & & $45(83.3)$ & \\
\hline $\mathrm{N+}$ & & $9(16.7)$ & \\
\hline Tumor location & n.a. & & n.a. \\
\hline Tongue & & $14(25.9)$ & \\
\hline Buccal & & $17(31.5)$ & \\
\hline Gum & & $11(20.4)$ & \\
\hline Lip & & $6(11.1)$ & \\
\hline Hard palate & & $3(5.5)$ & \\
\hline Mouth floor & & $2(3.7)$ & \\
\hline Retromolar & & $1(1.8)$ & \\
\hline
\end{tabular}

n.a.: not applicable; BMl: body mass index

The age, weight, and BMI are shown as the mean (standard deviation), other variables are shown as $\mathrm{n}(\%)$. Patients in Group 2 were significantly older.

The two groups were comparable in sex distribution, weight, and BMI. 
\#BMI was not normally distributed, shown data are medians and interquartile ranges.

Table 2. Comparison between linear delineation and threshold selection on C3 and masticatory muscle

\begin{tabular}{|c|c|c|c|c|c|c|}
\hline & \multicolumn{2}{|c|}{$\begin{array}{l}\text { Manual } \\
\text { Delineation }\end{array}$} & \multicolumn{2}{|c|}{$\begin{array}{l}\text { Threshold } \\
\text { Selection }\end{array}$} & \multirow[t]{2}{*}{$\begin{array}{l}\text { Diff } \\
(\%)\end{array}$} & \multirow[t]{2}{*}{$\begin{array}{l}p \text { - } \\
\text { value }\end{array}$} \\
\hline & Mean & SD & Mean & SD & & \\
\hline $\begin{array}{l}\text { Masticatory } \\
\text { muscles }\end{array}$ & 1379.4 & 419.4 & 1366.4 & 442.8 & $0.95 \%$ & 0.304 \\
\hline Masseter & 579.8 & 197.8 & 574.1 & 164.4 & $1.00 \%$ & 0.258 \\
\hline Pterygoid & 819.8 & 200.2 & 792.3 & 181.5 & $0.92 \%$ & 0.341 \\
\hline C3 muscles & 3074.9 & 1554.8 & 2866.4 & 1331.5 & $7.27 \%$ & 0.008 \\
\hline L3 muscles & 12603.5 & 2911.3 & 11884.2 & 2471.3 & $6.05 \%$ & 0.011 \\
\hline
\end{tabular}

Unit: $\mathrm{mm} 2$

Cross-sectional area of each muscle item includes the sum of the bilateral muscles.

SD: standard deviation;

Diff: Difference, defined as CSA of (Manual delineation - Threshold Selection)/Threshold selection* $100 \%$

Table 3. Image analysis between the patients with trauma and oral cavity cancer.

\begin{tabular}{llllll} 
Cross section area & Trauma & \multicolumn{3}{c}{ Oral cancer } & $p$-value* \\
& Mean & SD & Mean & SD & \\
\hline Masticatory muscles & 1512.2 & 544.5 & 1221.4 & 340.9 & 0.006 \\
\hline Masseter & 620.4 & 221.2 & 533.1 & 171.6 & 0.016 \\
\hline Pterygoid & 891.8 & 323.3 & 688.3 & 169.3 & $<0.001$ \\
\hline C3 muscles & 3034.2 & 1646.4 & 2701.2 & 1212.4 & 0.26 \\
\hline L3 muscles & 12589.4 & 4565.3 & 11239.1 & 2685.2 & 0.06
\end{tabular}

Unit: mm2; SD: standard deviation; CSA: cross-sectional area; PVM: paravertebral muscle;

SCM: sternocleidomastoid muscle. CSA of the masticatory muscles is defined as the sum of the CSAs of the bilateral skeletal muscles at the mandibular notch level, including the masseter and pterygoid muscles. Statistical significance is shown in bold. 
*All analyses are corrected for sex and age.

\section{Figures}

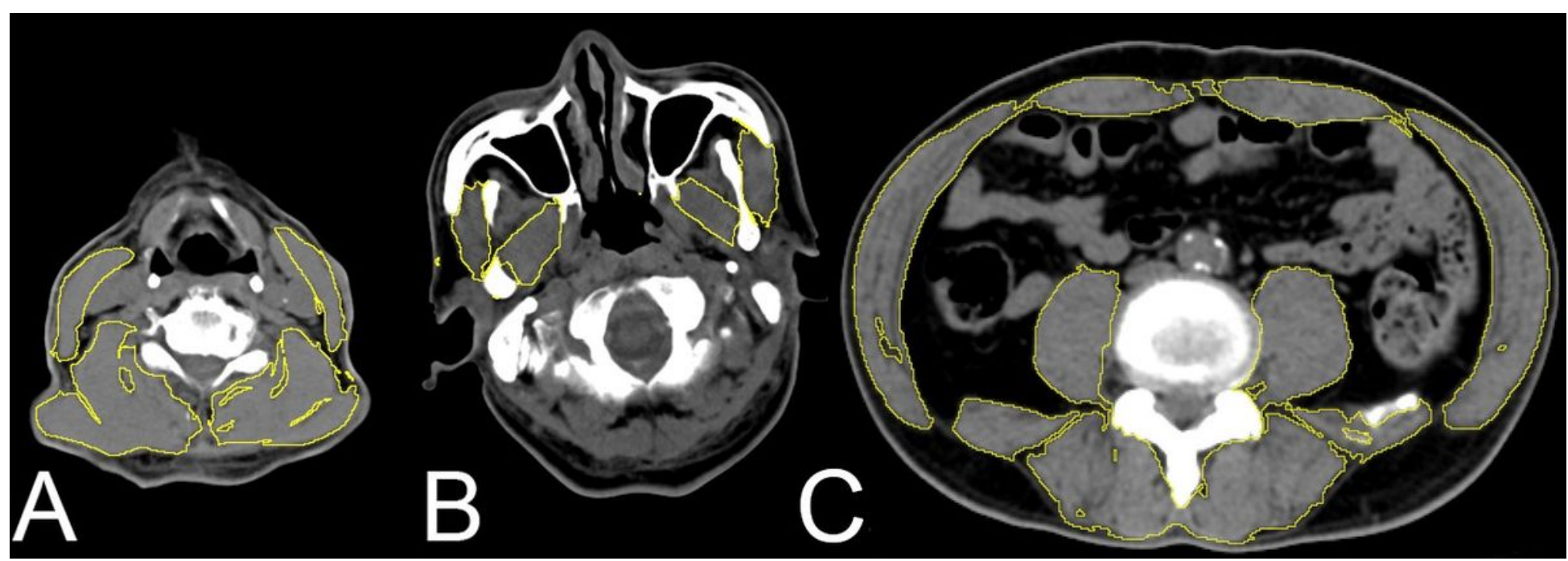

\section{Figure 1}

Examples of masticatory muscle, C3, and L3 delineation using Image J with a threshold setting for delineation from -29 to $+150 \mathrm{HU}$. (A) Delineations of the paravertebral muscles (PVM) and sternocleidomastoid muscles at the $\mathrm{C} 3$ level are delineated. (B) Delineations of the masseter and pterygoid muscles at the level of the mandibular notch. (C) Delineations of the L3 PVM.

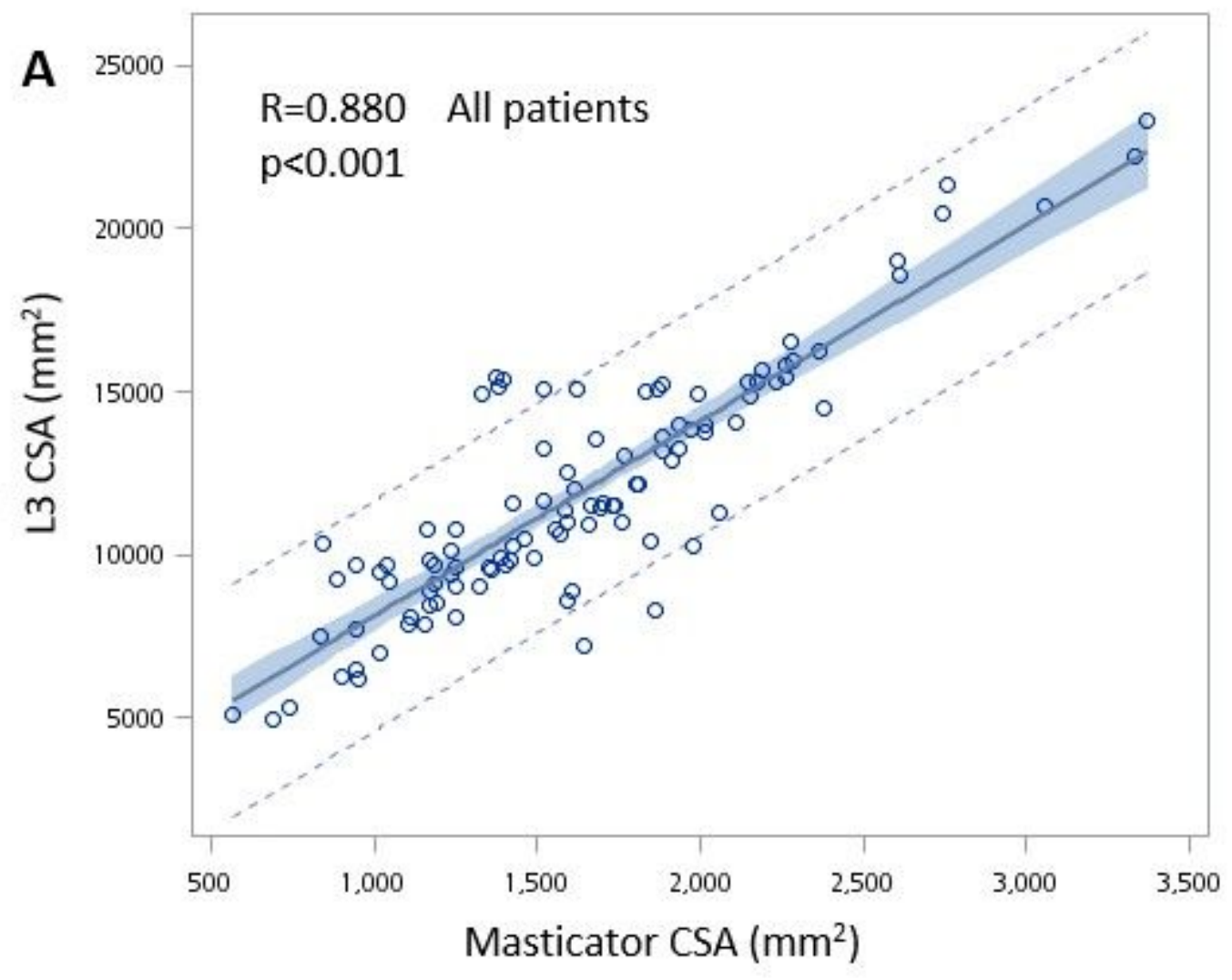




\section{Figure 2}

Linear regressions between the masticatory muscle cross-sectional areas with the L3 muscle crosssectional area. There was a significant correlation $(r=0.880, p<0.001)$ between the cross sectional area of the masticatory muscles at the mandibular notch level and the muscles at the L3 level. 